\title{
Review Article \\ Bone Health in Type 1 Diabetes: Where We Are Now and How We Should Proceed
}

\author{
Volha V. Zhukouskaya, ${ }^{1}$ Alla P. Shepelkevich, ${ }^{2}$ and Iacopo Chiodini' ${ }^{1}$ \\ ${ }^{1}$ Department of Clinical Sciences and Community Health, University of Milan, Unit of Endocrinology and Metabolic Disease, \\ Foundation IRCCS Cà Granda, Ospedale Maggiore Policlinico, Padiglione Granelli, F. Sforza Street, No. 35, 20122 Milan, Italy \\ ${ }^{2}$ Belarusian State Medical University, Dzerzinski Avenue, No. 83, 220116 Minsk, Belarus
}

Correspondence should be addressed to Volha V. Zhukouskaya; volha.zhukouskaya@gmail.com

Received 16 January 2014; Accepted 30 May 2014; Published 25 June 2014

Academic Editor: Qing-Sheng Mi

Copyright (C) 2014 Volha V. Zhukouskaya et al. This is an open access article distributed under the Creative Commons Attribution License, which permits unrestricted use, distribution, and reproduction in any medium, provided the original work is properly cited.

Type 1 diabetes (T1D) is autoimmune disease with chronic hyperglycaemic state. Besides diabetic retinopathy, nephropathy, and neuropathy, T1D is characterized by poor bone health. The reduced bone mineralization and quality/strength, due to hyperglycemia, hypoinsulinemia, autoimmune inflammation, low levels of insulin growth factor-1 (IGF-1), and vitamin D, lead to vertebral/hip fractures. Young age of T1D manifestation, chronic poor glycemic control, high daily insulin dose, low BMI, reduced renal function, and the presence of complications can be helpful in identifying T1D patients at risk of reduced bone mineral density. Although risk factors for fracture risk are still unknown, chronic poor glycemic control and presence of diabetic complications might raise the suspicion of elevated fracture risk in T1D. In the presence of the risk factors, the assessment of bone mineral density by dual-energy $\mathrm{X}$-ray absorptiometry and the search of asymptomatic vertebral fracture by lateral X-ray radiography of thorax-lumbar spine should be recommended. The improvement of glycemic control may have a beneficial effect on bone in T1D. Several experiments showed promising results on using anabolic pharmacological agents (recombinant IGF-1 and parathyroid hormone) in diabetic rodents with bone disorder. Randomized clinical trials are needed in order to test the possible use of bone anabolic therapies in humans with T1D.

\section{Introduction}

Type 1 diabetes (T1D) is an autoimmune disease that precipitates in genetically susceptible individuals by environmental factors. The body's own immune system attacks the betacells in the islets of Langerhans of the pancreas, destroying or damaging them sufficiently to reduce and eliminate insulin production, leading to the hypoinsulinemia and chronic hyperglycaemia [1]. T1D incidence has been globally rising during the past decades by as much as 3\% annually, the cause of which is unknown. If these trends will continue, the total prevalence of people with type 1 diabetes will increase in coming years [2].

Chronic hyperglycaemia in T1D leads, in course of time, to chronic complications. Besides acute diabetic complications, nowadays, health providers give more attention to the prevention of disabling chronic complications, such as diabetic retinopathy, nephropathy, neuropathy, and precocious atherosclerosis with early cardiovascular disease. Recently, a major interest has been focused on poor bone metabolism in T1D that can represent an overlooked complication of diabetes.

\section{What Do We Know? Bone Parameters and Fracture Risk in Type 1 Diabetes}

2.1. Bone Mineralization. An association between diabetes and reduced bone mass was firstly described by Albright and Reifenstein in 1948 [3]. In 1976 Levin and coauthors [4] demonstrated that almost $50 \%$ of patients with T1D had a reduction of bone mineral density (BMD) at the wrist. 
The studies concerning the bone metabolism in T1D can be categorized into two groups: (1) studies evaluating bone metabolism in diabetic children and adolescents who did not reach the peak of bone mass yet; (2) studies evaluating bone metabolism in adults who developed T1D after having reached peak of bone mass.

It should be admitted that it is rather difficult to study bone metabolism in such population as children/adolescents whose skeleton is still in the way of growing. Moreover, the majority of studies included the children/adolescents at different stages of puberty and, therefore, at different stages of acquisition of bone mass. This probably, has been one of the main reasons of the lack of concordant results about the impact of diabetes on growing bones. Some authors [5-11] showed no differences in BMD between T1D children/adolescents and their peers without diabetes. However, other authors found low bone mineral content (BMC) and low BMD both at spine and at femoral neck in T1D children/adolescents [12-18]. Moreover, some longitudinal studies $[6,9]$ demonstrated a significant reduction of either lumbar spine or femoral neck BMD in diabetic patients after 2-4 years of followup, despite normal BMD at baseline. Therefore, it seems that T1D, appeared in childhood, may alter the acquisition of bone mass that can be registered in youth ages or later in adult life.

Indeed, the majority of studies, performed on the T1D adults, consistently showed a reduction of BMD either at lumbar spine and/or at femur $[8,9,19-30]$. Only few studies [31-33], which were conducted on small groups of diabetic patients (less than 40 cases), were discordant. Vestergaard [34] having analyzed 80 studies regarding bone density in diabetes, has proved in his meta-analysis that T1D patients have lower BMD than the people without diabetes.

Frequency of reduced BMD in T1D varies largely from 3 to $40 \%[19,24,25,29,30]$. In our study by Eller-Vainicher and coauthors [35] about $30 \%$ of 175 T1D patients had low bone mass (osteopenia/osteoporosis) at spine and/or femur, which was significantly higher in comparison to healthy controls.

2.2. Geometric Bone Parameters. Another important observation about bone parameters in T1D, besides low bone mineralization, is the reduced bone size. Indeed, both studies on T1D animals [36-38] and T1D children/adolescents [10, $16,39,40]$ showed a significant decrease of bone crosssectional area at radius, tibias and femur and a decrease of femur cortical thickness, leading to smaller and thinner bones. Thus, the decrease in BMD measured by dual energy $\mathrm{X}$-ray absorptiometry (DEXA) could be due to the reduced bone size. On the contrary, Miazgowski et al. [29] did not find any differences in hip cross-section area between T1D adult males and healthy controls, in the presence, at the same time, of low BMD. Such discrepancy could be explained by the fact that reduced bone size in the phase of growing can normalize with age, as shown in a 5-year followup study by Bechtold et al. [39]. Nonetheless, lower bone size has been demonstrated even after completion of pubertal growth in T1D adolescents [40], and lower mineralization was found even in these smaller bones when measured with peripheral quantitative computer tomography (pQCT) [16].

\subsection{Bone Strength/Biomechanical Parameters and Bone Qual-} ity. Bone strength and bone quality play an important role in the bone health and contribute in the relevant manner to a fracture event.

Biomechanical parameters (maximum load, displacement, energy absorption capacity, stiffness, ultimate stress, toughness, and elastic modulus), measured with tensile test and nanoindentation, reflect how the bone is able to resist the applied load. In the studies [36-38], performed on the streptozotocin- (STZ-) induced diabetic animal models, a reduction in the whole bone strength (less toughness and more brittleness) has been observed, leading to increased susceptibility to even small energy load. On the other hand, Miazgowski et al. [29] has found only the tendency of crosssectional moment of inertia (CSMI) to be reduced in T1D adult men. Of note, in the latter study [29], CSMI was measured with DEXA, which might not be sensitive enough to evaluate biomechanical bone properties.

Few studies addressed the issue of bone quality in T1D. At one hand, STZ-induced diabetic animal models $[36,37]$ have shown changes of bone structure, analyzed with either microcomputed tomography $(\mu \mathrm{CT})$ and histomorphometry, such as lower bone volume and fewer and thinner trabeculae. At the same time, the sole human study [41] could not find any differences of bone structure between T1D patients and healthy controls. However, in the same study, T1D patients with fractures tended to have lower bone volume, trabecular thickness, and number and higher trabecular separation as compared to patients without fractures, therefore indicating possible microarchitectural deterioration in the fractured diabetic subjects [41]. Larger human studies are needed to prove whether bone strength and quality are altered in type 1 diabetes.

2.4. Fracture Risk. In T1D patients the frequency of lifetime fractures at any site has been reported to be increased as compared to counterparts without diabetes [27, 42]. The meta-analysis of Vestergaard [34] demonstrated a 6.94-fold increase risk of hip fracture in T1D. Further, in our study [43] T1D patients were found to have an increased prevalence of also asymptomatic vertebral fractures, which have been observed in $25 \%$ of diabetic subjects.

In conclusion, there is strong evidence that bones in T1D patients are characterized by poor mineralization and smaller and thinner size with reduced bone strength and quality, which can lead to a higher fracture incidence at any site, predominantly at femoral neck.

\section{Why and How Did It Happen? Pathophysiological Aspects of Bone Disorder in Type 1 Diabetes}

3.1. Bone Turnover: Osteoblastic and Osteoclastic Activity. Bone remodeling consists of bone formation and resorption, 
which are performed by osteoblasts and osteoclasts, respectively. Remodeling causes renewals to bone by removing old material with microcracks and consequently by constructing a new one. The coupling of these two processes represents a crucial moment in the maintenance of bone health. When the function of osteoblasts and/or osteoclasts is impaired, the bone apposition and resorption are altered, rendering bone remodeling inefficient to repair old material.

Remodeling is regulated by several hormones and cytokines, among which insulin, insulin-like growth factor1 (IGF-1), parathyroid hormone (PTH), thyroid hormones, cortisol, estrogen, vitamin D, and other cytokines of inflammation such as interleukin-1 and 6 (IL-1,6), tumor necrosis factor- $\alpha$ (TNF- $\alpha$ ), and transforming growth factor- $\beta 1$ (TGF$\beta 1)$ play a major role. The activity of osteoblasts and osteoclasts can be reflected by different markers measured both in the blood and in the urine. Bone formation markers consist of osteocalcin, bone-specific alkaline phosphatase, alkaline phosphatase, procollagen type 1 amino terminal propeptide, and procollagen type 1 carboxyl terminal propeptide, while resorptive markers consist of $\mathrm{N}$-terminal crosslinked telopeptide of type-I collagen, C-terminal cross-linked telopeptide of type-I collagen, tartrate-resistant acid phosphatase, pyridinoline, deoxypyridinoline, and hydroxyproline [44].

Osteocalcin is the most abundant noncollagenous protein of the bone matrix. It is a product of differentiated osteoblasts, and it promotes the recruitment and differentiation of circulating monocytes and osteoclasts precursors, suggesting its role on osteoblast-osteoclast interaction and bone resorption [45]. In the last decade particular interest has been addressed to the extraskeletal effects of the osteocalcin, one of which is glucose homeostasis. There is a reciprocal loop between osteoblast and pancreatic $\beta$-cells function. Circulating osteocalcin and, particularly, its undercarboxylated fraction (released during active bone resorption) exert a direct effect on $\beta$-cells, stimulating insulin production, and on adipocytes, enhancing adiponectin production. Adiponectin itself is able to promote insulin sensitivity. In turn, insulin also acts directly on osteoblasts and indirectly on osteoclasts. Locking the reciprocal loop, osteoclasts stimulate bone resorption with subsequent release of undercarboxylated osteocalcin in blood circulation [45].

Several studies demonstrated that bone metabolism in T1D is characterized by low bone turnover and, in particular, by reduced bone formation [44]. In T1D the osteoblast impairment is characterized by (1) decreased osteoblastogenesis; (2) low osteoblast differentiation; (3) low osteoblast activity (low levels of osteocalcin and reduced mineral apposition rate); (4) low osteoblast number (low osteoblast surface and osteoid surface); and (5) enhanced osteoblast death $[46,47]$. Additionally, slow and short osteoblastic cycle is accompanied by decreased osteoblast lineage selection due to impaired function of bone marrow stromal cells (BMSC) [48]. Osteoclast metabolism appears unaltered or decreased $[45,46]$.

At the molecular level, it is thought that inhibition of the Wnt/ $\beta$-catenin signaling and Runx 2 activity, which play significant role in the control of osteoblastogenesis and bone formation in physiological condition, is responsible for slowing down the osteoblastic metabolism $[47,49]$. However, the mechanisms leading to the inhibition of the Wnt/ $\beta$ catenin signaling and Runx 2 activity are still unknown.

It is possible that hyperglycemia, hypoinsulinemia, and autoimmune inflammation, well known characteristics of T1D, play a crucial role in impairing osteoblast differentiation and function. Moreover, the low levels of IGF-1 [9, 15, 47, 48, 50, 51] and vitamin D [52], which also usually accompany diabetes, may be an additional factor responsible of poor bone health (see Figure 1).

3.2. Role of Hyperglycemia: Oxidative Stress and AGEs. Hyperglycemia itself, regardless of its etiology, is detrimental for bone. Hyperglycemia may have a direct toxicity for osteoblasts, affecting the osteoblast signaling pathways [53] and may lead to an increased reactive oxygen species, induction of cellular osmotic responses, oxidative stress [53], and increased nonenzymatic glycosylation of proteins and DNA $[36,46]$. Nonenzymatic glycosylation during chronic hyperglycemic state and oxidative stress leads to the formation and deposition of advanced glycation end products (AGEs) in different tissues, including bone. AGEs, and in particular pentosidine, being one of the important products of nonenzymatic glycosylation, have been suggested to be deposited predominantly at skeletal sites with low bone turnover, as cortical bone [54], damaging in this way bone strength and quality [36]. This is thought to be the main mechanism of increased bone fragility and fractures, predominantly of long bones, especially femur, in T1D [34].

\subsection{Role of Hypoinsulinemia and Deficit of IGF-1. Research} over several decades has supported a primary role for insulin and IGF-1 in anabolic bone formation. Expression of insulin and IGF-1 receptors has been detected at different steps of osteoblast differentiation, from preosteoblast to mature ones [55]. Moreover, insulin and IGF-1 are important factors for osteoblast linage selection, since its receptors have been found also on osteogenic BMSC [48]. Insulin and IGF-1 utilize many of the same cellular proteins to achieve various cellular outcomes. In addition, they are able to cross-talk with two major proosteogenic pathways that ultimately regulate Runx2 activity in osteoblasts, such as the canonical Wnt/ $\beta$-catenin signaling and the bone mineral protein- (BMP-) 2 pathways [55].

As it has been already mentioned before, T1D is characterized by hypoinsulinemia and also by an IGF-1 decrease $[9,15,47,48,50,51,55]$. The reduction of IGF-1 levels in T1D is not fully explained. It has been hypothesized that both hyperglycemia and the state of chronic inflammation, through enhanced expression of proteins "suppressors of cytokine signaling" (SOCS), can suppress the growth hormone $(\mathrm{GH})$ activity with subsequent reduction of IGF-1 synthesis $[50,51$, 56].

Beside the insulin and IGF-1 levels reduction, recent studies on diabetic rodents $[47,48]$ have found a decreased expression of insulin and IGF-1 receptors and of some 


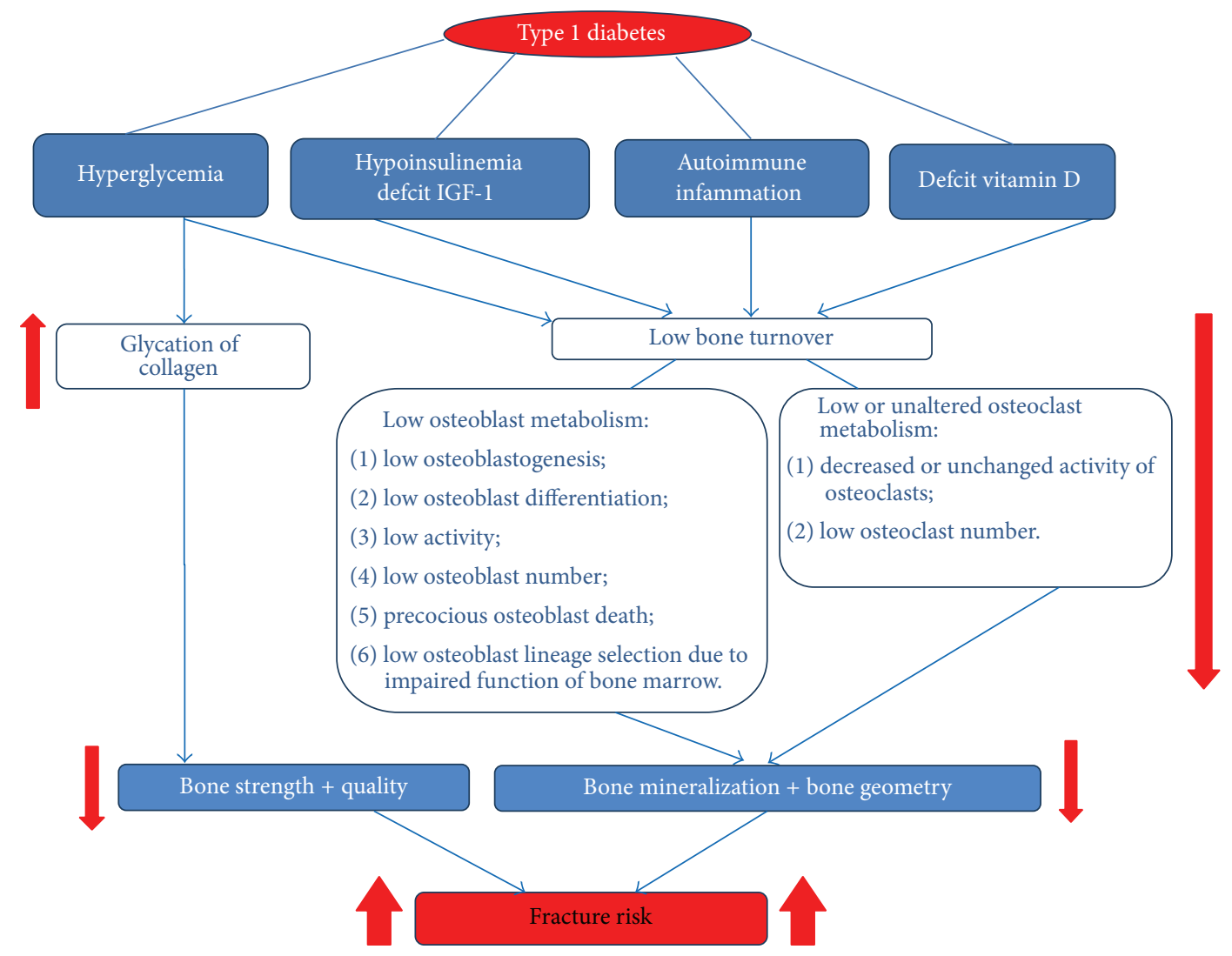

FIGURE 1: Pathophysiological aspects of bone disorder in type 1 diabetes.

important proteins for the insulin and IGF-1 signaling both in osteoblasts and in osteogenic BMSCs.

Therefore, the presence of hypoinsulinemia, IGF-1 reduction, and of an altered signaling of these molecules can impair both osteoblastic function and the osteogenic potential of BMSCs, leading to reduced bone formation.

3.4. Role of Autoimmune Inflammation. Autoimmune inflammatory state is one of pathogenic characteristics of T1D. In humans, some studies indicate no inflammation while others indicate higher intracellular TNF- $\alpha$ in CD8+ $\mathrm{T}$ cells at the time of diagnosis and higher intracellular TNF- $\alpha$ in CD $4+$ T lymphocytes in patients at 3 months after diagnosis [46]. TNF- $\alpha$ is well known to activate osteoclast bone resorption and decrease bone formation. In fact, animal models showed that the increased expression of TNF- $\alpha$, both in bone and bone marrow, leads to osteoblastic disfunction and its precocious death. Treatment with TNF- $\alpha$ neutralizing antibodies reduces diabetes-induced increases in osteoblast apoptosis [57, 58]. Moreover, inflammatory cytokines induce enhanced expression of adipogenic genes (peroxisome proliferator-activated receptor $\gamma 2$ : $\operatorname{PAAR} \gamma 2$ ), which, in turn, switches the differentiation of stem cells from osteoblasts to adipocytes, determining bone marrow adiposity [59, 60], in this way, leading to an altered mesenchymal cell lineage selection (adipogenesis at the cost of osteoblastogenesis) and to the reduction of bone formation.
However, this is only animal models, and there are no human studies so far. Therefore, this topic about the link between autoimmune inflammation and bone damage in T1D still remains less explored.

3.5. Role of Vitamin D Deficit. Vitamin D plays an important role in the bone health. Its active form $1,25(\mathrm{OH})_{2} \mathrm{D}$ interacts with its vitamin $\mathrm{D}$ nuclear receptor, which is present in the small intestine, kidneys, and other tissues. It stimulates intestinal calcium absorption and calcium reabsorption from the glomerular filtrate. $1,25(\mathrm{OH})_{2} \mathrm{D}$ interacts with its vitamin $\mathrm{D}$ receptor in the osteoblast, stimulating the expression of receptor activator of nuclear factor $B$ ligand (RANKL). This, in turn, interacts with receptor activator of nuclear factor B (RANK) to induce immature monocytes to become mature osteoclasts, which dissolve the matrix and mobilize calcium and other minerals from the skeleton [61]. Nutritional rickets in children and osteomalacia in adults are undisputed consequences of vitamin $\mathrm{D}$ deficiency.

Vitamin D insufficiency/deficiency in T1D is a common finding in most [52] but not all studies [52]. However, vitamin $\mathrm{D}$ insufficiency was also frequent in nondiabetic individuals [52]. The exact diabetes-specific mechanism contributing to vitamin D deficiency is not clear. However, some authors hypothesized a role of the different genetic variants of vitamin $\mathrm{D}$ receptor, $1 \alpha$-hydroxylase, and other genes of vitamin $\mathrm{D}$ metabolism involved in vitamin $\mathrm{D}$ transport, cholesterol 
TABLE 1: Clinical risk factors associated with poor bone health in T1D.

Clinical risk factors for low bone mineralization

Clinical risk factors for fracture risk

(1) Young age of T1D manifestation

(2) Poor glycemic control

(3) Presence of diabetic complications

(4) Daily insulin dose $>0.67 \mathrm{U} / \mathrm{kg}$

(5) $\mathrm{BMI}<23.5 \mathrm{~kg} / \mathrm{m}^{2}$

(6) Renal function $<88.8 \mathrm{~mL} / \mathrm{min}$

T1D: type 1 diabetes; BMD: bone mineral density; BMI: body mass index.

synthesis, and vitamin D hydroxylation [52]. Moreover, exaggerated urinary loss of vitamin D-binding protein in T1D patients, particularly in those with microalbuminuria, might contribute mechanistically to vitamin D deficiency in this disease $[62,63]$.

\section{What Should We Do in Clinical Practice? Management of Type 1 Diabetic Patients at Risk of Bone Disorder}

4.1. Who Is at Risk of Bone Disorder? Clinical Risk Factors Associated with Poor Bone Health in Type 1 Diabetes. when evaluating T1D patients in clinical practice, it is very important to give the answers to the following questions: who is at the risk of bone disorder and who should be evaluated for it? In order to respond correctly to these questions, adequate algorithms, including clinical factors able to reflect poor bone health in T1D, should be developed.

Clinical factors associated with poor bone health in T1D can be divided into two groups (Table 1): (1) factors associated with low BMD; (2) factors associated with fractures.

4.1.1. Clinical Factors Associated with Low BMD. The age of diabetes manifestation, disease duration, glycosylated hemoglobin (HbAlc), diabetic complications, daily insulin dose, BMI, and renal function can give information about the possible presence of low BMD in T1D.

The age of T1D manifestation may be crucial for the acquisition of bone mass. Although data about bone mineralization in children/adolescents are inconsistent, some authors $[6,9]$ have demonstrated a significant reduction of either lumbar spine or femoral neck BMD in diabetic patients after 2-4 years of followup, even having showed normal BMD at baseline. Moreover, early manifestation of T1D can be a risk factor for smaller bone size $[10,16,39,40]$. Therefore, a young age of T1D occurrence may be considered a risk factor for low BMD in T1D patients.

On the other hand, the majority of studies have found no association between low BMD and duration of T1D [5-9, 12, $15,29,34,35,42,64]$.

On the basis of the data regarding the effect of hyperglycemia on osteoblast, one could expect to find an association between BMD and glycometabolic control as reflected by HbAlc. However, only few studies have found the link between poor glycemic control and low BMD [13, 14, 27, 65], probably, because HbAlc was evaluated not only during the last three months but also during the previous years of disease. On the other hand, the lack of a correlation between BMD and HbAlc may also depend on a nonlinear relationship between these variables, hardly detectable by the classic statistics. In our study [35], we have applied a special mathematic approach, such as artificial neural network (ANN). In this study we found that HbAlc was connected with low BMD through the link with the diabetes complications (see Figure 2). Indeed, the diabetic complications are the result of the chronic exposure to high blood glucose of target organs and the finding of an association between chronic complications and low BMD may also reflect the effect of chronic hyperglycemia on bone.

The chronic diabetes complications per se have been suggested to predict low BMD in T1D. The reduced visual function and the presence of diabetic neuropathy may predispose patients to low physical activity, which, in turn, may cause bone loss $[7,19,22,24]$. The presence of diabetic nephropathy with negative calcium balance and reduced vitamin $\mathrm{D}$ level was reported to be an early indicator of osteopenia in T1D $[19,22]$.

Insulin is considered an anabolic agent for bone [55], and, therefore, one should expect BMD to increase with the increase of daily insulin dose. On the contrary, in the study by Eller-Vainicher et al. [35] and in the study by Léger et al. [15], patients with diabetes with low BMD had higher insulin dose. This finding could be explained by the following hypothesizes. Firstly, it is possible that the need of high insulin dose may reflect the presence of a more severe disease (i.e., a more pronounced inflammatory milieu), leading per se to bone damage. In keeping, a direct correlation between insulin dose and HbAlc [35] and between levels of inflammation markers/oxidative stress and HbAlc [14] has been demonstrated. This hypothesis is supported by the ANN analysis (Figure 2), [35], showing that insulin dose was strictly connected with HbAlc and then with low BMD, although through diabetes complications. Secondly, higher insulin demands might simply reflect higher insulin resistance and higher autoimmune inflammation at the level of all the tissues, including bone. Indeed, recently it has been suggested that in T1D, insulin resistance raises the insulin demands, leading to the beta-cell stress. In this setting autoimmunity may be a secondary accelerator operating in those with particular HLA genotype [66].

Beside all the factors described above, some studies [34, $35,42]$ have reported low body mass index (BMI) [35] to be associated with low $\mathrm{BMD}$, pointing to the importance 


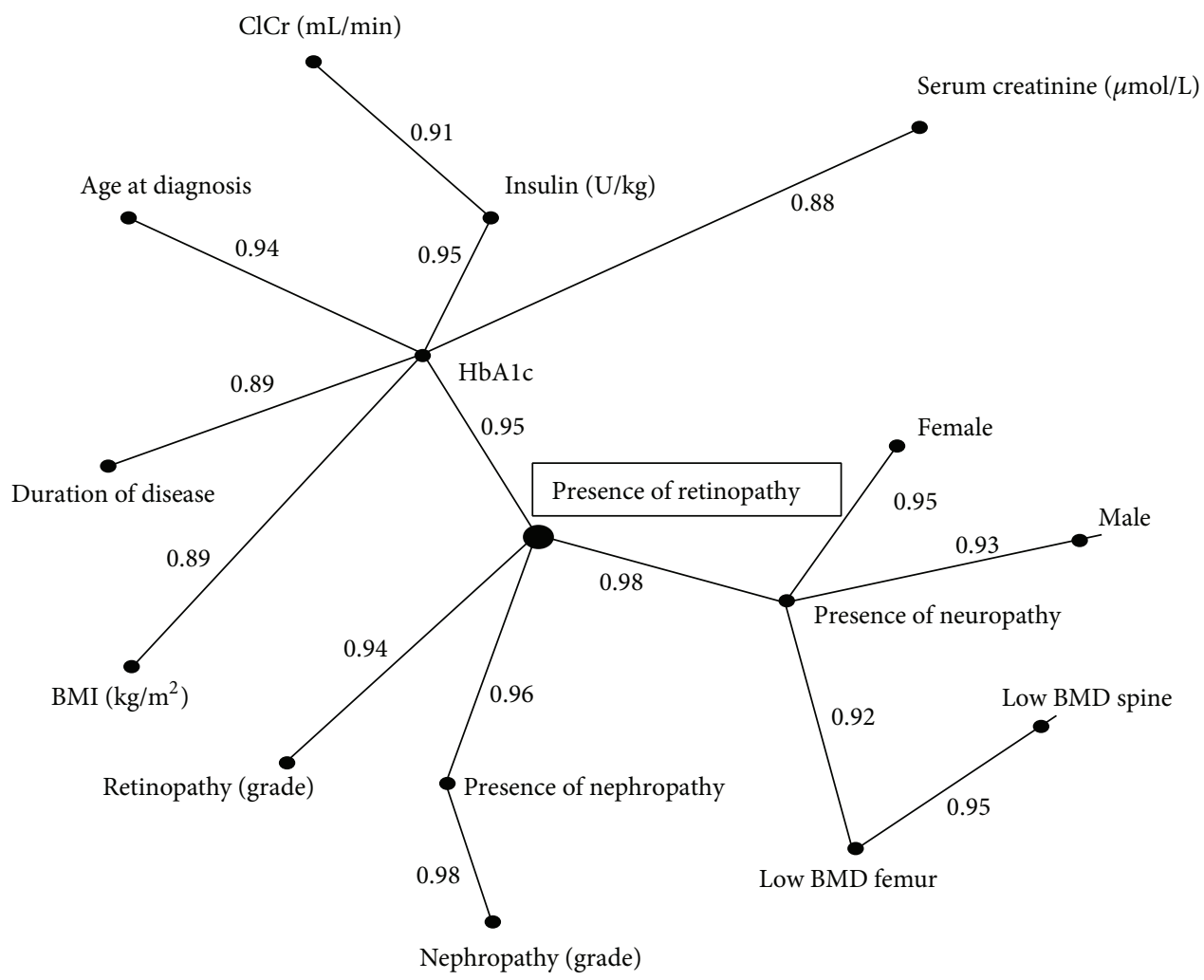

FIGURE 2: Semantic connectivity map of studied variables. The figures on the connections represent the strength of the link on a $0-1$ scale. Low BMD femur, presence of low F-BMD; low BMD spine, presence of low LS-BMD. Males and females are both connected to neuropathy but with a different conditional probability. The link for females is stronger (0.95) than it is for males (0.93). BMD: bone mineral density; F: femur; LS: lumbar spine; BMI: body mass index; ClCr: clearance creatinine; HbAlc: glycosylated hemoglobin. (Diabetes care by American Diabetes Association Reproduced with permission of American Diabetes Association in the format Journal/magazine via Copyright Clearance Center. Order Detail ID: 64182026).

of maintaining of lean mass and weight in type 1 diabetic patients.

Finally, kidney function seems to be important for femoral BMD not only in general population [67], but also in T1D population [35].

Interestingly, Eller-Vainicher and coauthors [35] has found the thresholds for daily insulin dose, BMI, and renal function $\left(>0.67 \mathrm{U} / \mathrm{kg},<23.5 \mathrm{~kg} / \mathrm{m}^{2}\right.$, and $<88.8 \mathrm{~mL} / \mathrm{min}$, resp.), below which $\mathrm{T} 1 \mathrm{D}$ patients may be at risk of poor bone mineralization. In the absence of these risk factors the probability to have normal $\mathrm{BMD}$ is $84.2 \%$ and measuring BMD may not be necessary. On the contrary, in the presence of all these risk factors the probability of low BMD is $62.9 \%$ and the measurement of BMD might be considered.

4.1.2. Clinical Factors Associated with Fractures. Besides $\mathrm{BMD}$, the other BMD-independent clinical factors associated with fractures have not been well studied. However, one can hypothesize that HbAlc and diabetic complications, being the result of high blood glucose levels, may be associated with fractures in T1D.

Although low bone mass is a common finding in T1D, it seems that low BMD is of poor fracture prediction in this kind of patients $[34,43]$, as it happens in other several forms of secondary osteoporosis [68]. T1D patients may have fractures even in the presence of normal BMD values [34, 43]. This fact emphasizes the presence of poor bone quality/strength, besides low bone mineralization, in T1D. On the other hand, Zhukouskaya et al. [43], having analyzed asymptomatic morphometric vertebral fractures in T1D population, have shown that the more severe vertebral fractures were associated with low lumbar spine BMD, which underlines that BMD still remains to be crucial for fracture event.

Since the elevated fracture risk in T1D seems to be related to reduced bone quality and strength rather than to reduced bone mass and AGEs (the effect of the chronic hyperglycemia) are the main responsible of low bone quality, the association between fractures and HbAlc should be logical. Nevertheless, only in one study [42] clinical fractures were associated with HbAlc, while the majority of studies have not shown any association between these two variables $[34,43]$. This could be explained by the fact that HbAlc, if measured only at the beginning and/or at the end of followup, cannot reliably mirror the glycometabolic control over time.

The diabetic complications per se have been suggested to contribute little to the overall risk of fractures in diabetes $[43,69]$, However, in our study [43] T1D patients with vertebral fracture tended to have higher prevalence of diabetic 
complications, especially retinopathy and neuropathy. Since low BMD is associated with the presence of complications and low lumbar spine BMD is associated with fractures, the diabetic complications might not be a BMD-independent fracture risk factor in T1D.

In our study we tried to define an algorithm for individuating the T1D patients to be screened for bone damage. In summary, in T1D patients with the diabetic complications (retinopathy, nephropathy, and neuropathy) and/or with daily insulin dose $>0.67 \mathrm{U} / \mathrm{kg}, \mathrm{BMI}<23.5 \mathrm{~kg} / \mathrm{m}^{2}$, and renal function $<88.8 \mathrm{~mL} / \mathrm{min}$, the screening for the bone disorder (low bone mineralization + high fracture risk) should be recommended.

\subsection{Management of Type 1 Diabetic Patients at Risk of Bone} Disorder. There is still no consensus on the correct evaluation and management of T1D patients at risk of bone disorder. However, we propose the following measures which are necessary to be done in these patients (Figure 3 ).

In order to exclude other possible causes of secondary osteoporosis some laboratory tests should be performed including [70] (1) general exams (blood cell count, serum protein electrophoresis, C-reactive protein, liver function with glutamic oxaloacetic transaminase (GOT), glutamic pyruvic transaminase (GPT), $\gamma$-glutamyltransferase $(\gamma$-GT), and renal function with creatinine); (2) mineral metabolism (total serum calcium corrected for albumin, serum phosphate, alkaline phosphatase (ALP), 25-hydroxyvitamin D (25OHD), and 24-hour urinary calcium); (3) thyroid and, in men, testes function (thyroid stimulating hormone, TSH, and total testosterone). Moreover, the possible presence of an associated celiac disease should be excluded in selected patients by performing antiendomysial and antitransglutaminase antibodies. Further laboratory tests may be required, depending on comorbidities and clinical findings.

In order to assess bone mineralization and the presence of vertebral fractures, a DXA evaluation at lumbar spine and at femoral neck and lateral X-ray radiography of thorax-lumbar spine or DXA combined with vertebral fracture assessment (VFA) should be performed [70] in the presence of diabetic complications and/or high daily insulin dose, low BMI, and reduced renal function.

\subsection{Treatment of Type 1 Diabetes-Associated Bone Disorder.} The best approach to treat patients with T1D related bone disorder is still not clear. On the basis of the pathogenesis of the bone disorder in T1D, the following strategies may lead to the improvement of poor bone health: (1) restoration of hypoinsulinemia and glycometabolic control; (2) reduction of autoimmune inflammation; (3) restoration of low levels of IGF-1; and (4) restoration of low levels of vitamin D.

Since hypoinsulinemia and hyperglycemia play an important role in damaging bone, insulin treatment accompanied by reduction of glycaemia seems to be the pivotal point in treatment and prevention of bone disorder in T1D. In the prospective study of Campos Pastor et al. [22], although the statistical significance was not reached, a BMD increase was associated with the improvement of glycemic control in T1D patients on intensive insulin treatment after 7 years of followup. However, the insulin treatment with reduction of hyperglycemia, probably, is not enough for bone health, since an elevated fracture risk is still present in T1D even after initiation of intensive insulin treatment. This may be due to several reasons. Firstly, insulin treatment is beneficial for bone mineralization, but it is not sufficient for the restoration of bone quality/strength [38]. Secondly, in order to avoid the risk of hypoglycemia it is not possible to reduce glycaemia to values of subjects without diabetes. Therefore, even a slight chronic hyperglycemia may be sufficient for damaging bone. Finally, the other additional factors besides hyperglycemia (i.e., autoimmune inflammation, deficit of IGF-1, and vitamin D), interfering with the bone health in T1D, are probably scarce or not influenced by the correction of the glycometabolic control.

Recently several experiments on animal models have been focused on the reduction of autoimmune inflammation and on the treatment with recombinant IGF-1 (rhIGF-1), in order to improve bone mineralization and quality in T1D. Treatment with TNF- $\alpha$-specific inhibitors reduces diabetesinduced increases in osteoblast apoptosis [46]. Fowlkes et al. [55] has shown favorable effect of rhIGF-1 in promoting new bone formation and in improving of bone biomechanical properties in STZ-induced diabetic rodents. To date, however, no studies are available on the possible therapeutical use of TNF- $\alpha$-specific inhibitors and rhIGF-1 in humans with T1D.

The efficacy of vitamin D on T1D related bone damage has been examined only minimally in animal models. In the STZinduced rat model of T1D, low femoral BMD has improved significantly after treatment with $1 \alpha$-hydroxyvitamin D3 [52]. Clinical trials are needed to investigate the role that vitamin $\mathrm{D}$ status may play in the intervention or reversal of bone damage in humans with T1D.

As T1D related osteoporosis is characterized by a reduced bone apposition and osteoblast differentiation and function, the anabolic therapy with PTH seems to be an interesting option. Motyl et al. [71] have studied effect of PTH treatment in STZ-induced diabetic rodents, showing increasing of bone mineralization by promoting remodeling and reducing diabetes-induced osteoblast apoptosis and making the conclusion that intermittent PTH therapy might be an option to promote bone formation and resorption, which are both depressed in diabetic patients. To date, however, no data on humans are available on the possible usefulness of PTH anabolic therapy in T1D patients.

Finally, weight-bearing physical activity has been recently demonstrated to have a positive effect on bone mineral acquisition in children with T1D, similarly to what happen in children without T1D [72].

Due to the lack of data on the possible therapeutic options on humans, most recommendations that can be given nowadays to the T1D patients at risk and with manifested bone disorder are derived from the good clinical practice and from the experience of the physician rather than from evidence-based guidelines.

Intensive insulin treatment, being a standard treatment of T1D, with improvement of glycemic control should be 


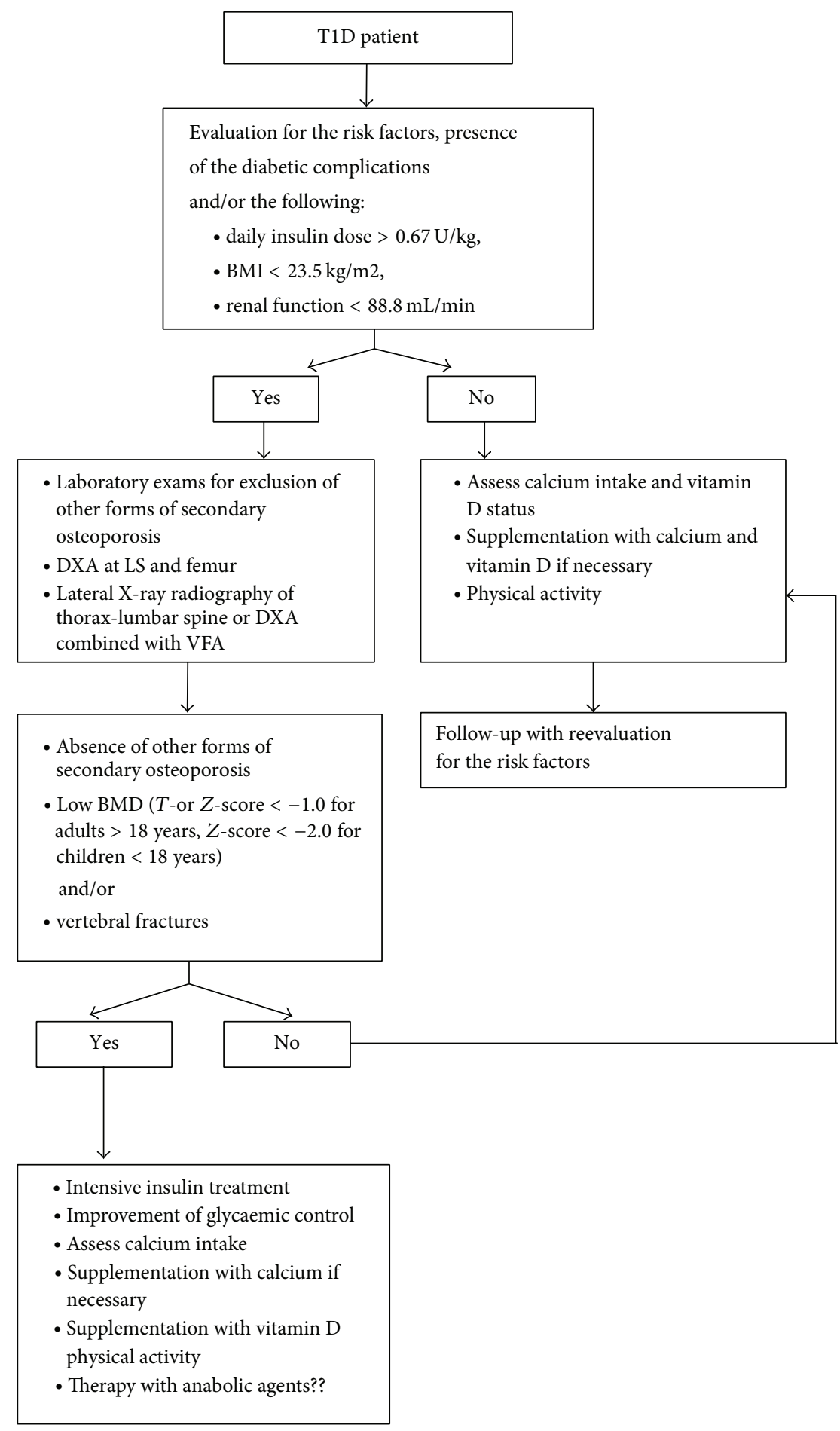

BMD: bone mineral density;

T1D: type 1 diabetes;

DXA: dual-energy X-ray absorptiometry;

LS: lumbar spine;

VFA: vertebral fracture assessment;

BMI: body mass index.

FIGURE 3: Flow-chat for evaluation, management, and treatment of T1D patients at risk of bone disorder. 
taken in consideration in all patients. Insulin with reduction of hyperglycemia would be beneficial not only for bone but also for prevention of chronic diabetic complications. Annual screening for microalbuminuria, annual ophthalmologic exam, and annual testing for pressure and vibration sensation should be performed in order to reach early diagnosis of diabetic nephropathy, retinopathy, and neuropathy, respectively.

Supplementation with calcium and vitamin D should be advised to the T1D patients with bone disorder. A daily uptake of $1200 \mathrm{mg}$ calcium is generally required, ideally through the diet, but supplementation can be used if dietary uptake is inadequate. According to the guidelines regarding prevention and treatment of vitamin D deficiency [61], vitamin D deficient subjects should be supplemented with vitamin D3 at dose of 600-1000 U/day for children and 1500-2000 U/day for adults.

Weight-bearing sports, including ball games, jumping activities, or gymnastics should be encouraged in T1D children to optimize bone mineral acquisition during growth and potentially prevent the development of osteoporosis later in life [72].

At the end of this chapter we propose a flow-chat (Figure 3) for evaluation, management, and treatment of T1D patients at risk and with manifested bone disorder.

\section{Which Way Should We Proceed? Conclusion and Future Prospects}

In summary, T1D is characterized by poor bone health, which should be recognized as a diabetic complication among the other well-known complications such as retinopathy, nephropathy, and neuropathy. Slow bone turnover is the main characteristic of T1D-associated bone disorder, which leads to reduced mineralization and reduced quality and strength with consequent fracture event as the most important clinical manifestation. Although during last decade many studies both on animals and humans have been focused on the pathogenesis of T1D related bone damage and on the risk factors for the identification of T1D patients at risk of bone disorder, several questions still remain to be answered.

Firstly, since BMD represents a poor clinical tool for fracture prediction, as it often happens in case of secondary osteoporosis [68], we need to develop some methods, easyto-perform in clinical practice, able to predict fracture risk in T1D patients. Trabecular bone score (TBS), being indirect measure of bone quality [73] and easily obtainable through DXA, has been shown to predict better than BMD the fracture risk in patients with some forms of secondary osteoporosis $[68,74,75]$. Therefore, prospective studies are needed to investigate the usefulness of TBS in prediction of fracture risk in T1D.

Secondly, it is possible that good glycemic control may exert a beneficial effect on bone, but it is not clear how strict we should maintain glycemic control and below which level we should lower HbAlc in order to prevent or improve bone disorder in T1D. Therefore, we need prospective studies focused on the changes of bone metabolism/mineralization/fracture risk after intensification of insulin treatment (e.g., through insulin pump), which is known to lead to a notable improvement of glycemic control.

Finally, it is not clear yet what kind of drugs should be used in T1D patients with manifested bone disorder, who do not improve with only good glycemic control and supplementation of calcium/vitamin D and who, probably, need pharmacological intervention. Some promising results seem to come from the use of anabolic pharmacological agents (rhIGF-1 and PTH) in diabetic rodents with bone disorder. Therefore, randomized clinical trials are needed in order to understand whether it could be the case in humans.

\section{Conflict of Interests}

The authors declare that there is no conflict of interests regarding the publication of this paper.

\section{References}

[1] T. L. van Belle, K. T. Coppieters, and M. G. von Herrath, "Type 1 diabetes: etiology, immunology, and therapeutic strategies," Physiological Reviews, vol. 91, no. 1, pp. 79-118, 2011.

[2] L. Guariguata, "Estimating the worldwide burden of type 1 diabetes," Diabetes Voice, vol. 56, no. 2, pp. 6-8, 2011.

[3] F. Albright and E. C. Reifenstein, "Bone development in diabetic children: a roentgen study," The American Journal of the Medical Sciences, vol. 174, pp. 313-319, 1948.

[4] M. E. Levin, V. C. Boisseau, and L. V. Avioli, "Effects of diabetes mellitus on bone mass in juvenile and adult onset diabetes," The New England Journal of Medicine, vol. 294, no. 5, pp. 241-245, 1976.

[5] J. Pascual, J. Argente, M. B. Lopez et al., "Bone mineral density in children and adolescents with diabetes mellitus type 1 of recent onset," Calcified Tissue International, vol. 62, no. 1, pp. 31-35, 1998.

[6] A. Salvatoni, G. Mancassola, R. Biasoli et al., "Bone mineral density in diabetic children and adolescents: a follow-up study," Bone, vol. 34, no. 5, pp. 900-904, 2004.

[7] F. R. Brandao, E. J. Vicente, C. H. Daltro, M. Sacramento, A. Moreira, and L. Adan, "Bone metabolism is linked to disease duration and metabolic control in type 1 diabetes mellitus," Diabetes Research and Clinical Practice, vol. 78, no. 3, pp. 334339, 2007.

[8] E. Y. Liu, J. Wactawski-Wende, R. P. Donahue, J. Dmochowski, K. M. Hovey, and T. Quattrin, "Does low bone mineral density start in post-teenage years in women with type 1 diabetes?" Diabetes Care, vol. 26, no. 8, pp. 2365-2369, 2003.

[9] L. D. Mastrandrea, J. Wactawski-Wende, R. P. Donahue, K. M. Hovey, A. Clark, and T. Quattrin, "Young women with type 1 diabetes have lower bone mineral density that persists over time," Diabetes Care, vol. 31, no. 9, pp. 1729-1735, 2008.

[10] S. Bechtold, I. Dirlenbach, K. Raile, V. Noelle, W. Bonfig, and H. P. Schwarz, "Early manifestation of type 1 diabetes in children is a risk factor for changed bone geometry: data using peripheral quantitative computed tomography," Pediatrics, vol. 118, no. 3, pp. e627-e634, 2006.

[11] A. B. R. Maggio, S. Ferrari, M. Kraenzlin et al., "Decreased bone turnover in children and adolescents with well controlled type 1 diabetes," Journal of Pediatric Endocrinology and Metabolism, vol. 23, no. 7, pp. 697-707, 2010. 
[12] P. Gunczler, R. Lanes, V. Paz-Martinez et al., "Decreased lumbar spine bone mass and low bone turnover in children and adolescents with insulin dependent diabetes mellitus followed longitudinally," Journal of Pediatric Endocrinology and Metabolism, vol. 11, no. 3, pp. 413-419, 1998.

[13] G. Valerio, A. del Puente, A. Esposito-del Puente, P. Buono, E. Mozzillo, and A. Franzese, "The lumbar bone mineral density is affected by long-term poor metabolic control in adolescents with type 1 diabetes mellitus," Hormone Research, vol. 58, no. 6 , pp. 266-272, 2002.

[14] K. Heilman, M. Zilmer, K. Zilmer, and V. Tillmann, "Lower bone mineral density in children with type 1 diabetes is associated with poor glycemic control and higher serum ICAM1 and urinary isoprostane levels," Journal of Bone and Mineral Metabolism, vol. 27, no. 5, pp. 598-604, 2009.

[15] J. Léger, D. Marinovic, C. Alberti et al., "Lower bone mineral content in children with type 1 diabetes mellitus is linked to female sex, low insulin-like growth factor type I levels, and high insulin requirement," Journal of Clinical Endocrinology and Metabolism, vol. 91, no. 10, pp. 3947-3953, 2006.

[16] M. T. Saha, H. Sievänen, M. K. Salo, S. Tulokas, and H. H. Saha, "Bone mass and structure in adolescents with type 1 diabetes compared to healthy peers," Osteoporosis International, vol. 20, no. 8, pp. 1401-1406, 2009.

[17] J. Heap, M. A. Murray, S. C. Miller, T. Jalili, and L. J. MoyerMileur, "Alterations in bone characteristics associated with glycemic control in adolescents with type 1 diabetes mellitus," Journal of Pediatrics, vol. 144, no. 1, pp. 56-62, 2004.

[18] E. A. Hamed, N. H. Abu Faddan, H. A. Adb Elhafeez, and D. Sayed, "Parathormone-25(OH)-vitamin D axis and bone status in children and adolescents with type 1 diabetes mellitus," Pediatric Diabetes, vol. 12, no. 6, pp. 536-546, 2011.

[19] M. Muñoz-Torres, E. Jódar, F. Escobar-Jiménez, P. J. LópezIbarra, and J. D. Luna, "Bone mineral density measured by dual X-ray absorptiometry in Spanish patients with insulindependent diabetes mellitus," Calcified Tissue International, vol. 58, no. 5, pp. 316-319, 1996.

[20] M. Rix, H. Andreassen, and P. Eskildsen, "Impact of peripheral neuropathy on bone density in patients with type 1 diabetes," Diabetes Care, vol. 22, no. 5, pp. 827-831, 1999.

[21] J. T. Tuominen, O. Impivaara, P. Puukka, and T. Rönnemaa, "Bone mineral density in patients with type 1 and type 2 diabetes," Diabetes Care, vol. 22, no. 7, pp. 1196-1200, 1999.

[22] M. M. Campos Pastor, P. J. López-Ibarra, F. Escobar-Jiménez, M. D. Serrano Pardo, and A. García-Cervigón, "Intensive insulin therapy and bone mineral density in type 1 diabetes mellitus: a prospective study," Osteoporosis International, vol. 11, no. 5, pp. 455-459, 2000.

[23] S. A. G. Kemink, A. R. M. M. Hermus, L. M. J. W. Swinkels, J. A. Lutterman, and A. G. H. Smals, "Osteopenia in insulindependent diabetes mellitus: prevalence and aspects of pathophysiology," Journal of Endocrinological Investigation, vol. 23, no. 5, pp. 295-303, 2000.

[24] A. Rozadilla, J. M. Nolla, E. Montana et al., "Bone mineral density in patients with type 1 diabetes mellitus," Joint Bone Spine, vol. 67, no. 3, pp. 215-218, 2000.

[25] P.-J. López-Ibarra, M. M. C. Pastor, F. Escobar-Jiménez et al., "Bone mineral density at time of clinical diagnosis of adultonset type 1 diabetes mellitus," Endocrine Practice, vol. 7, no. 5, pp. 346-351, 2001.

[26] D. J. Hadjidakis, A. E. Raptis, M. Sfakianakis, A. Mylonakis, and S. A. Raptis, "Bone mineral density of both genders in type 1 diabetes according to bone composition," Journal of Diabetes and its Complications, vol. 20, no. 5, pp. 302-307, 2006.

[27] K. K. Danielson, M. E. Elliott, T. Lecaire, N. Binkley, and M. Palta, "Poor glycemic control is associated with low BMD detected in premenopausal women with type 1 diabetes," Osteoporosis International, vol. 20, no. 6, pp. 923-933, 2009.

[28] E. J. Hamilton, V. Rakic, W. A. Davis et al., "Prevalence and predictors of osteopenia and osteoporosis in adults with type 1 diabetes," Diabetic Medicine, vol. 26, no. 1, pp. 45-52, 2009.

[29] T. Miazgowski, S. Pynka, M. Noworyta-Zietara, B. Kryzanowska-Świniarska, and R. Pikul, "Bone mineral density and hip structural analysis in type 1 diabetic men," European Journal of Endocrinology, vol. 156, no. 1, pp. 123-127, 2007.

[30] O. Alexopoulou, J. Jamart, J. P. Devogelaer, S. Brichard, P. de Nayer, and M. Buysschaert, "Bone density and markers of bone remodeling in type 1 male diabetic patients," Diabetes and Metabolism, vol. 32, no. 5, pp. 453-458, 2006.

[31] G. Hampson, C. Evans, R. J. Petitt et al., "Bone mineral density, collagen type $1 \alpha 1$ genotypes and bone turnover in premenopausal women with diabetes mellitus," Diabetologia, vol. 41, no. 11, pp. 1314-1320, 1998.

[32] C.-M. Ingberg, M. Palmér, J. Åman, B. Arvidsson, E. Schvarcz, and C. Berne, "Body composition and bone mineral density in long-standing type 1 diabetes," Journal of Internal Medicine, vol. 255, no. 3, pp. 392-398, 2004.

[33] M. J. Bridges, S. H. Moochhala, J. Barbour, and C. A. Kelly, "Influence of diabetes on peripheral bone mineral density in men: a controlled study," Acta Diabetologica, vol. 42, no. 2, pp. 82-86, 2005.

[34] P. Vestergaard, "Discrepancies in bone mineral density and fracture risk in patients with type 1 and type 2 diabetes-a metaanalysis," Osteoporosis International, vol. 18, no. 4, pp. 427-444, 2007.

[35] C. Eller-Vainicher, V. V. Zhukouskaya, Y. V. Tolkachev et al., "Low bone mineral density and its predictors in type 1 diabetic patients evaluated by the classic statistics and artificial neural network analysis," Diabetes Care, vol. 34, no. 10, pp. 2186-2191, 2011.

[36] M. J. Silva, M. D. Brodt, M. A. Lynch et al., "Type 1 diabetes in young rats leads to progressive trabecular bone loss, cessation of cortical bone growth, and diminished whole bone strength and fatigue life," Journal of Bone and Mineral Research, vol. 24, no. 9, pp. 1618-1627, 2009.

[37] J. S. Nyman, J. L. Even, C. Jo et al., "Increasing duration of type 1 diabetes perturbs the strength-structure relationship and increases brittleness of bone," Bone, vol. 48, no. 4, pp. 733-740, 2011.

[38] N. Erdal, S. Gürgül, C. Demirel, and A. Yildiz, "The effect of insulin therapy on biomechanical deterioration of bone in streptozotocin (STZ)-induced type 1 diabetes mellitus in rats," Diabetes Research and Clinical Practice, vol. 97, no. 3, pp. 461467, 2012.

[39] S. Bechtold, S. Putzker, W. Bonfig, O. Fuchs, I. Dirlenbach, and H. P. Schwarz, "Bone size normalizes with age in children and adolescents with type 1 diabetes," Diabetes Care, vol. 30, no. 8, pp. 2046-2050, 2007.

[40] I. Roggen, I. Gies, J. Vanbesien, O. Louis, and J. de Schepper, "Trabecular bone mineral density and bone geometry of the distal radius at completion of pubertal growth in childhood type 1 diabetes," Hormone Research in Paediatrics, vol. 79, no. 2, pp. 68-74, 2013. 
[41] L. A. G. Armas, M. P. Akhter, A. Drincic, and R. R. Recker, "Trabecular bone histomorphometry in humans with type 1 diabetes mellitus," Bone, vol. 50, no. 1, pp. 91-96, 2012.

[42] T. Neumann, A. Sämann, S. Lodes et al., "Glycaemic control is positively associated with prevalent fractures but not with bone mineral density in patients with typel diabetes," Diabetic Medicine, vol. 28, no. 7, pp. 872-875, 2011.

[43] V. V. Zhukouskaya, C. Eller-Vainicher, V. V. Vadzianava et al., "Prevalence of morphometric vertebral fractures in patients with type 1 diabetes," Diabetes Care, vol. 36, no. 6, pp. 1635-1640, 2013.

[44] J. Starup-Linde, "Diabetes, biochemical markers of bone turnover, diabetes control and bone," Frontiers in Endocrinology, vol. 4, article 21, 2013.

[45] A. Patti, L. Gennari, D. Merlotti, F. Dotta, and R. Nuti, "Endocrine actions of osteocalcin," International Journal of Endocrinology, vol. 2013, Article ID 846480, 10 pages, 2013.

[46] L. R. McCabe, J. Zhang, and S. Raehtz, "Understanding the skeletal pathology of type 1 and 2 diabetes mellitus," Critical Reviews in Eukaryotic Gene Expression, vol. 21, no. 2, pp. 187206, 2011.

[47] M. Hie, N. Iitsuka, T. Otsuka, and I. Tsukamoto, "Insulindependent diabetes mellitus decreases osteoblastogenesis associated with the inhibition of Wnt signaling through increased expression of Sost and Dkk1 and inhibition of Akt activation," International Journal of Molecular Medicine, vol. 28, no. 3, pp. 455-462, 2011.

[48] Y. F. Zhao, D. L. Zeng, L. G. Xia et al., "Osteogenic potential of bone marrow stromal cells derived from streptozotocininduced diabetic rats," International Journal of Molecular Medicine, vol. 31, no. 3, pp. 614-620, 2013.

[49] S. Portal-Núñez, D. Lozano, L. Fernández de Castro, A. R. de Gortázar, X. Nogués, and P. Esbrit, "Alterations of the Wnt/ $\beta$ catenin pathway and its target genes for the $\mathrm{N}$ - and $\mathrm{C}$-terminal domains of parathyroid hormone-related protein in bone from diabetic mice," FEBS Letters, vol. 584, no. 14, pp. 3095-3100, 2010.

[50] L. J. Moyer-Mileur, H. Slater, K. C. Jordan, and M. A. Murray, "IGF-1 and IGF-binding proteins and bone mass, geometry, and strength: relation to metabolic control in adolescent girls with type 1 diabetes," Journal of Bone and Mineral Research, vol. 23, no. 12, pp. 1884-1891, 2008.

[51] B. J. van Sickle, J. Simmons, R. Hall, M. Raines, K. Ness, and A. Spagnoli, "Increased circulating IL- 8 is associated with reduced IGF-1 and related to poor metabolic control in adolescents with type 1 diabetes mellitus," Cytokine, vol. 48, no. 3, pp. 290-294, 2009.

[52] K. M. Thrailkill and J. L. Fowlkes, "The role of vitamin d in the metabolic homeostasis of diabetic bone," Clinical Reviews in Bone and Mineral Metabolism, vol. 11, no. 1, pp. 28-37, 2013.

[53] S. Botolin and L. R. McCabe, "Chronic hyperglycemia modulates osteoblast gene expression through osmotic and nonosmotic pathways," Journal of Cellular Biochemistry, vol. 99, no. 2, pp. 411-424, 2006.

[54] P. Odetti, S. Rossi, F. Monacelli et al., "Advanced glycation end products and bone loss during aging," Annals of the New York Academy of Sciences, vol. 1043, pp. 710-717, 2005.

[55] J. L. Fowlkes, R. C. Bunn, and K. M. Thrailkill, "Contributions of the insulin/insulin-like growth factor-1 axis to diabetic osteopathy," Journal of Diabetes and Metabolism, vol. 1, no. 3, p. S1-003, 2011.
[56] S. F. Ahmed and C. Farquharson, "The effect of GH and IGF1 on linear growth and skeletal development and their modulation by SOCS proteins," Journal of Endocrinology, vol. 206, no. 3, pp. 249-259, 2010.

[57] L. M. Coe, R. Irwin, D. Lippner, and L. R. McCabe, "The bone marrow microenvironment contributes to type I diabetes induced osteoblast death," Journal of Cellular Physiology, vol. 226, no. 2, pp. 477-483, 2011.

[58] K. J. Motyl, S. Botolin, R. Irwin et al., "Bone inflammation and altered gene expression with type Idiabetes early onset," Journal of Cellular Physiology, vol. 218, no. 3, pp. 575-583, 2009.

[59] S. Botolin, M. Faugere, H. Malluche, M. Orth, R. Meyer, and L. R. McCabe, "Increased bone adiposity and peroxisomal proliferator-activated receptor- $\gamma 2$ expression in type I diabetic mice," Endocrinology, vol. 146, no. 8, pp. 3622-3631, 2005.

[60] S. Botolin and L. R. McCabe, "Bone loss and increased bone adiposity in spontaneous and pharmacologically induced diabetic mice," Endocrinology, vol. 148, no. 1, pp. 198-205, 2007.

[61] M. F. Holick, N. C. Binkley, H. A. Bischoff-Ferrari et al., "Evaluation, treatment, and prevention of vitamin D deficiency: an endocrine society clinical practice guideline," Journal of Clinical Endocrinology and Metabolism, vol. 96, no. 7, pp. 19111930, 2011.

[62] K. M. Thrailkill, T. Nimmo, R. C. Bunn et al., "Microalbuminuria in type 1 diabetes is associated with enhanced excretion of the endocytic multiligand receptors megalin and cubilin," Diabetes Care, vol. 32, no. 7, pp. 1266-1268, 2009.

[63] K. M. Thrailkill, C. Jo, G. E. Cockrell, C. S. Moreau, and J. L. Fowlkes, "Enhanced excretion of vitamin D binding protein in type 1 diabetes: a role in vitamin D deficiency?" Journal of Clinical Endocrinology and Metabolism, vol. 96, no. 1, pp. 142149, 2011.

[64] A. Joshi, P. Varthakavi, M. Chadha, and N. Bhagwat, "A study of bone mineral density and its determinants in type 1 diabetes mellitus," Journal of Osteoporosis, vol. 2013, Article ID 397814, 8 pages, 2013.

[65] M. O. Çamurdan, P. Ciaz, A. Bideci, and F. Demirel, "Role of hemoglobin Alc, duration and puberty on bone mineral density in diabetic children," Pediatrics International, vol. 49, no. 5, pp. 645-651, 2007.

[66] T. J. Wilkin, "Is autoimmunity or insulin resistance the primary driver of type 1 diabetes?" Current Diabetes Reports, vol. 13, no. 5, pp. 651-656, 2013.

[67] S. A. Jamal, V. J. D. Swan, J. P. Brown et al., "Kidney function and rate of bone loss at the hip and spine: the Canadian multicentre osteoporosis study," The American Journal of Kidney Diseases, vol. 55, no. 2, pp. 291-299, 2010.

[68] V. Morelli, C. Eller-Vainicher, A. S. Salcuni et al., "Risk of new vertebral fractures in patients with adrenal incidentaloma with and without subclinical hypercortisolism: a multicenter longitudinal study," Journal of Bone and Mineral Research, vol. 26, no. 8, pp. 1816-1821, 2011.

[69] P. Vestergaard, L. Rejnmark, and L. Mosekilde, "Diabetes and its complications and their relationship with risk of fractures in type 1 and 2 diabetes," Calcified Tissue International, vol. 84, no. 1, pp. 45-55, 2009.

[70] S. Ferrari, M. L. Bianchi, J. A. Eisman et al., "Osteoporosis in young adults: pathophysiology, diagnosis, and management," Osteoporosis International, vol. 23, no. 12, pp. 2735-2748, 2012.

[71] K. J. Motyl, L. K. McCauley, and L. R. McCabe, "Amelioration of type I diabetes-induced osteoporosis by parathyroid hormone 
is associated with improved osteoblast survival," Journal of Cellular Physiology, vol. 227, no. 4, pp. 1326-1334, 2012.

[72] A. B. R. Maggio, R. R. Rizzoli, L. M. Marchand, S. Ferrari, M. Beghetti, and N. J. Farpour-Lambert, "Physical activity increases bone mineral density in children with type 1 diabetes," Medicine and Science in Sports and Exercise, vol. 44, no. 7, pp. 1206-1211, 2012.

[73] D. Hans, N. Barthe, S. Boutroy, L. Pothuaud, R. Winzenrieth, and M. Krieg, "Correlations between trabecular bone score, measured using anteroposterior dual-energy X-ray absorptiometry acquisition, and 3-dimensional parameters of bone microarchitecture: an experimental study on human cadaver vertebrae," Journal of Clinical Densitometry, vol. 14, no. 3, pp. 302-312, 2011.

[74] W. D. Leslie, B. Aubry-Rozier, O. Lamy, and D. Hans, “TBS (trabecular bone score) and diabetes-related fracture risk," Journal of Clinical Endocrinology and Metabolism, vol. 98, no. 2, pp. 602-609, 2013.

[75] C. Eller-Vainicher, V. Morelli, F. M. Ulivieri et al., "Bone quality, as measured by trabecular bone score in patients with adrenal incidentalomas with and without subclinical hypercortisolism," Journal of Bone and Mineral Research, vol. 27, no. 10, pp. 22232230, 2012. 


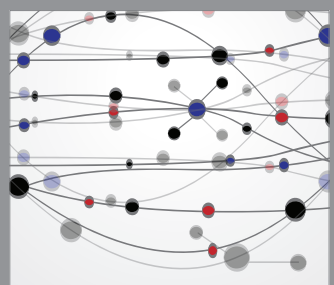

The Scientific World Journal
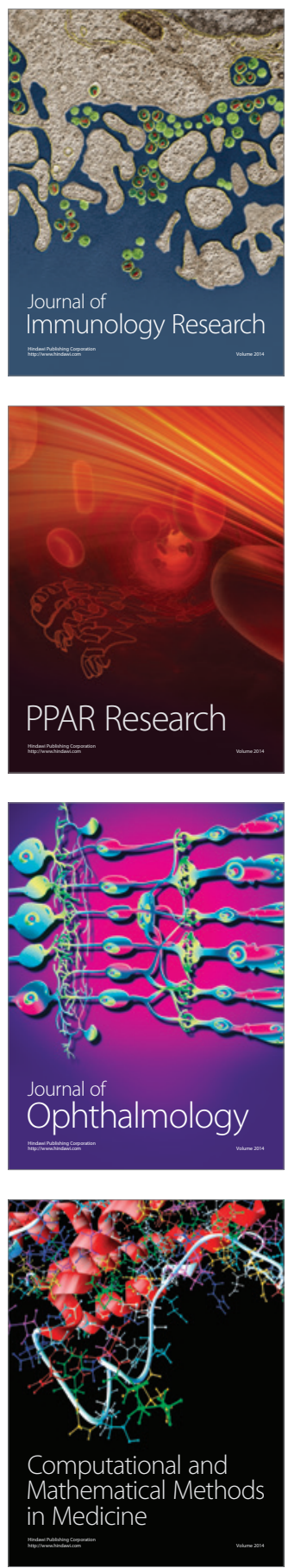

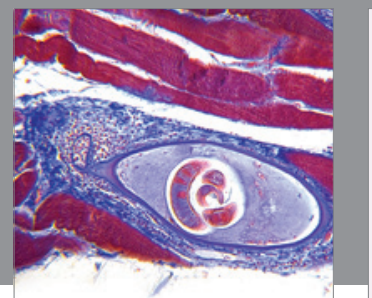

Gastroenterology

Research and Practice
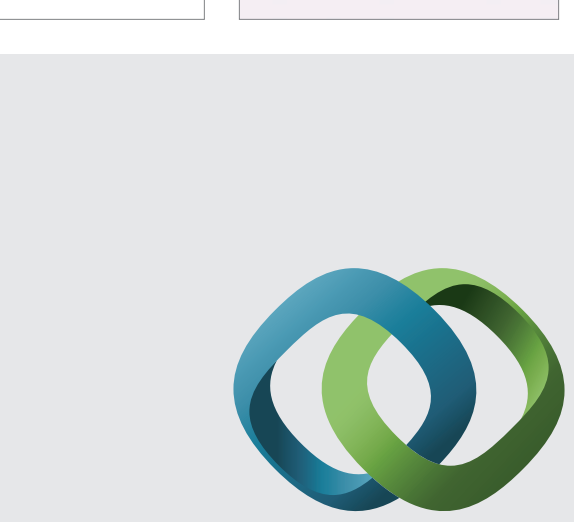

\section{Hindawi}

Submit your manuscripts at

http://www.hindawi.com
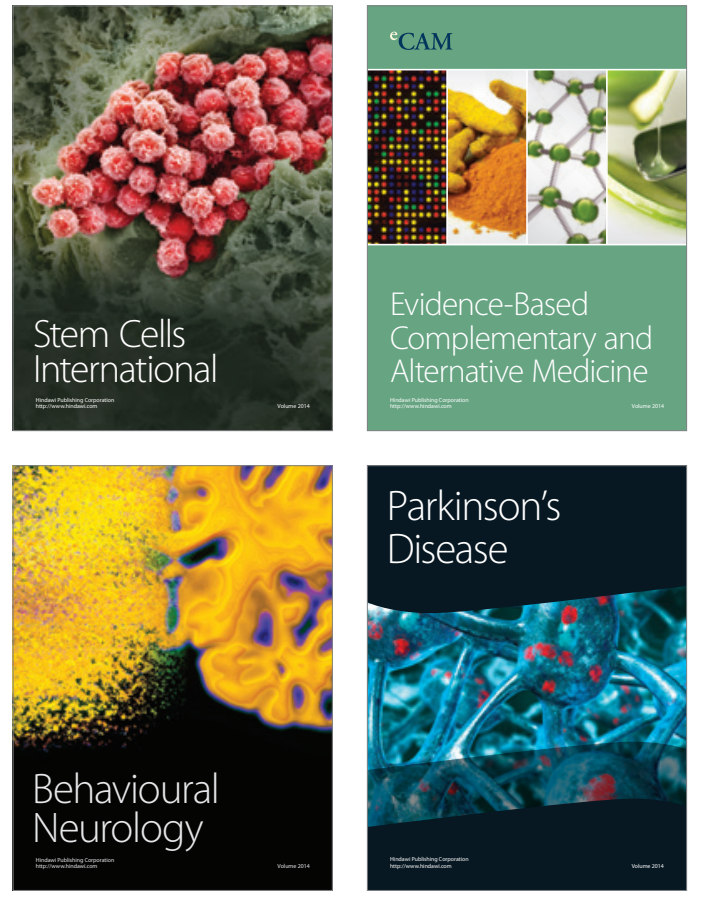
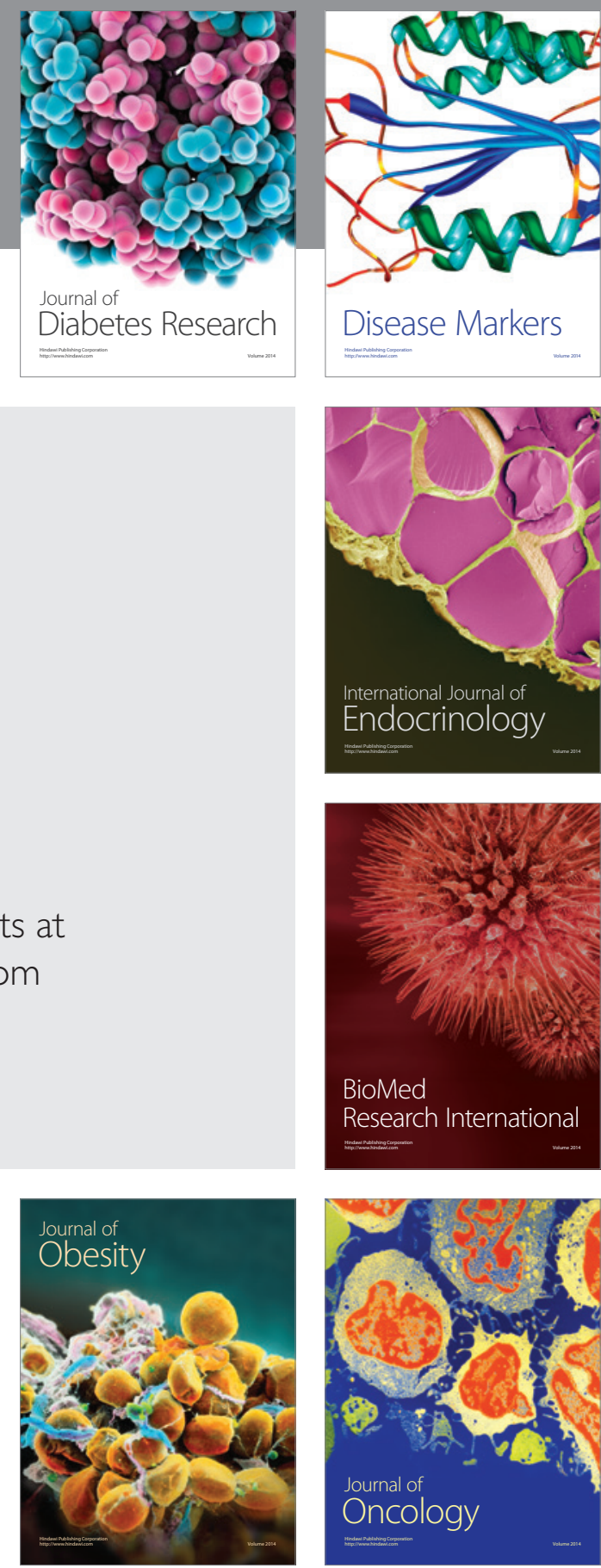

Disease Markers
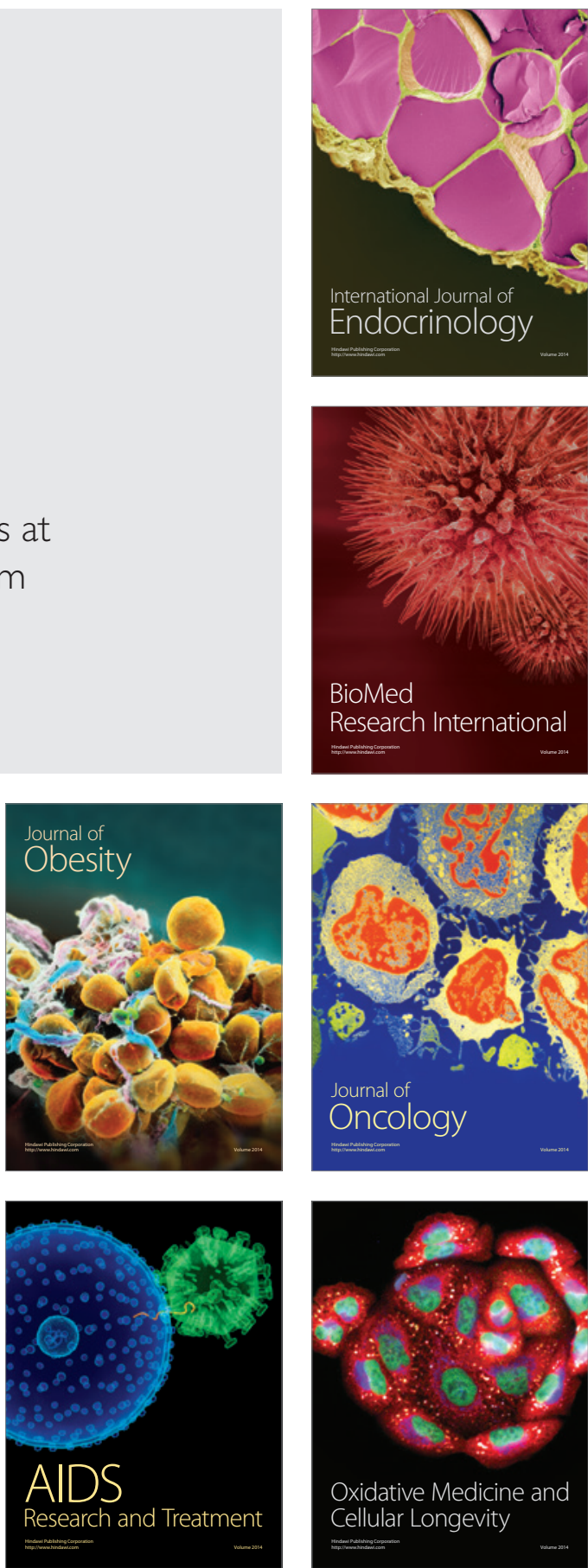